This paper has been officially published in Comptes Rendus Mécanique, Vol. 335, pp. 455-460, 2007 (DOI : 10.1016/j.crme.2007.05.023).

\title{
Fracture of rigid solids: a discrete approach based on a damaging interface modelling.
}

Claire SILVANI (silvani@lma.cnrs-mrs.fr), Université de Provence \& LMA (UPR 7051-CNRS), 31 chemin Joseph Aiguier, 13402 Marseille cedex 20, France.

Thierry DÉSOYER (thierry.desoyer@ec-marseille.fr), EC-Marseille \& LMA (UPR 7051-CNRS), Technopôle de Château-Gombert, 38 rue Joliot Curie, 13451 Marseille Cedex 20, France.

Stéphane BONELLI (stephane.bonelli@aix.cemagref.fr), Cemagref, 3275 route de Cézanne, CS 40061, 13182 Aix-en-Provence cedex 5, France.

\begin{abstract}
We describe the progressive and delayed fracture of rigid solids by a discrete modelling. Each rigid solid is considered as an assembly of particles with initial cohesive bonds, the latter decreasing progressively during the loading. A damaging interface model is proposed to describe this progressive phenomenon. The model has been implemented in a discrete element code. The first illustrative example, which is actually a parametric study, deals with the progressive damage and sudden fracture of a single interface submitted to an uniaxial tension. The second example is related to the crushing of an assembly of rigid solids $-i$. e. a granular medium - submitted to an œdometric compression.

Keywords: Granular medium; rigid solids; interfaces; damage; fracture.

Résumé Nous décrivons la rupture progressive et différée de solides rigides par une approche discrète. Chaque solide rigide est représenté par une collection de particules, initialement liées par une cohésion qui peut progressivement diminuer au cours du chargement. Un modèle d'endommagement interfacial est proposé pour décrire cette décroissance progressive. Implémenté dans un code de calcul par éléments discrets, ce modèle permet de simuler la rupture différée de collections de solides rigides. Le premier exemple illustratif, qui est en fait une étude paramétrique, est relatif à l'endommagement progressif puis la rupture d'une unique interface soumise à une traction simple. Le second exemple porte sur la rupture et l'attrition d'une collection de solides rigides $-i$. e. d'un milieu granulaire - sous compression œdométrique. Mots-clés : Milieu granulaire ; solides rigides ; interfaces ; endommagement ; rupture
\end{abstract}

\section{Introduction}

The general frame of this study is that of the progressive (finite cracking velocity) and delayed (with respect to the loading) fracture of rigid solids interacting by contact and friction. An illustrative example of such a structural problem is this of a rockfill dam, which can globally settle due to the local fracture of rock blocks in the time, see e. g. Deluzarche and Cambou, [1]; Oldecop and Alonso, [2].

Choice is here made to get numerically approximated solutions of the contact-friction part of the problem by using the discrete element method proposed by Jean and Moreau (see $e . g$. [3], [4] ). However, due to the fact that the rigid solids (or grains) - which will be all assumed of the same characteristic size $D^{S}-$ can break, each of them is considered as an assembly of rigid particles - which will be also all assumed of the same characteristic size $D^{p} \ll D^{S}$. These particles are assumed to be initially 'glued'. From a numerical point of view, a grain, i. e. an assembly of rigid particles, must thus be seen as a mesh of the rigid solid, in which a crack can initiate (resp. propagate) only on (resp. through) the contact zones between rigid grains. Consequently, from a physical point of view, these contact zones have to be considered as rigid but breakable interfaces.

Strong cohesive forces are supposed to exist initially on the interfaces (see $e . g$. Delenne et al, [5]), giving to them their initial tensile strength. It is then assumed that, when a given interface $I$ - characteristic 
area $S \approx\left(D^{p}\right)^{2}$ - is submitted to a sufficiently strong tensile force, microcracks and/or microcavities, $i$. e. damage, initiate, grow and, eventually, coalesce, that leads to the fracture of the interface (and so, to the irreversible vanishing of the cohesive forces).

Section 2 of this paper is devoted to the presentation of a thermodynamically consistent damaging interface model where, in agreement with the general frame of this study, the evolution of the damage is at the same times progressive and delayed. Two illustrative examples are presented in Section 3. The first one is that of a single interface between two particles submitted to an uniaxial tensile force: the analytical solution is given, from which a parametric study of the damaging interface model is done. The second example is related to the crushing of an assembly of two-dimensional rigid solids $-i$. e. a two-dimensional granular medium - due to an œedometric compression: the results here presented have been obtained using a numerical code in which the damaging interface model has been implemented.

\section{A damaging interface modelling}

The (thermo)dynamic system considered in this section is an interface $I$ between two grains. Like the grains, $I$ is assumed to be rigid : the area of the surface $S$ occupied by $I$ is then constant, whatever the forces acting on are. Furthermore, the displacement jump $[\boldsymbol{u}]$ through $S$ is assumed to be zero whenever $I$ is not destroyed ( $i$. e. whenever $S$ is clearly defined) ; consequently, $[\boldsymbol{u}]$ cannot be considered as a state variable of $I$. Actually, only one 'mechanical' state variable will be considered there, denoted by $d$ (scalar) and characterizing the damage by microcracking and/or microcavitation of the constitutive material of $I$. It will be assumed that $d \in\left[0, \frac{1}{m}\right]$ where $m>0$ is a material parameter whose physical meaning will be discussed later on. It must be here emphasized that, as soon as $d=1 / m, I$ is destroyed and the contactfriction interactions between the both grains have to be considered on the basis of the Signorini-Coulomb equations (see $e . g$. [4]), which will not be detailed in the present paper.

The damaging interface model is actually based on previous works on continuum damage mechanics by Marigo, [6], where the necessary and sufficient condition for the intrinsic dissipation to be non negative is simply given by $\dot{d} \geq 0$. Denoting by $\sigma$ the stresses acting in $S$, assumption is then made that $\sigma$ is homogeneous. On the other hand, it is assumed that, due to the damage, the effective tough surface of $I$ is not $S$ but its only undamaged part $(1-m d) S$. Consequently, the stresses are simply linked to the global force $\boldsymbol{F}$ (defined in such a way that $F_{N}=\boldsymbol{F} . \boldsymbol{N}>0$ when $I$ is submitted to a tensile force) by:

$$
\boldsymbol{F}=(1-m d) S \boldsymbol{\sigma} \cdot \boldsymbol{N}
$$

A damage yield surface is next introduced. Once more, it is clearly inspired by the works by Marigo, [6]. However, for a sake of consistency between the present interfacial damage model and the CoulombSignorini one (see also Cangemi et al, [7]), which must 'merge' in the latter one as soon as $d=1 / m$, the damage yield surface is here expressed as a function of $F_{N}$ and $\boldsymbol{F}_{\boldsymbol{t}}=\boldsymbol{F}-F_{N} \boldsymbol{N}, i$. e.:

$$
g^{d}\left(F_{N}, \boldsymbol{F}_{\boldsymbol{t}}, d\right)=F_{N}+\frac{1}{\mu}\left|\boldsymbol{F}_{\boldsymbol{t}}\right|-F_{0}^{d}(1-m d)=0
$$

where $\mu$ is the friction coefficient between the both grains when $I$ is destroyed $(d=1 / m), F_{0}^{d}>0$ the damage yield when $d=0$, and $m>0$ a 'softening' parameter (the greater $m$, the stronger the softening). As previously indicated, Eqn. (2) reduces to the classical Coulomb's yield surface as soon as $d=1 / \mathrm{m}$. As for the fracture of $I$, which can occur suddenly when $I$ is sufficiently damaged, it is controlled by a fracture yield surface, which reads:

$$
g^{f}\left(F_{N}, \boldsymbol{F}_{\boldsymbol{t}}, d\right)=F_{N}+\frac{1}{\mu}\left|\boldsymbol{F}_{\boldsymbol{t}}\right|-F_{0}^{f}(1-m d)=0
$$

where $F_{0}^{f} \geq F_{0}^{d}$ is the maximal tensile force $I$ can undergo. It must be here emphasized that mechanical states $\left(F_{N}, \boldsymbol{F}_{\boldsymbol{t}}, d\right)$ such that $g^{f}\left(F_{N}, \boldsymbol{F}_{\boldsymbol{t}}, d\right)>0$ cannot be reached $-i$. e., as soon as $g^{f}\left(F_{N}, \boldsymbol{F}_{\boldsymbol{t}}, d\right)=0, I$ is destroyed - and that, whatever the reachable mechanical state $\left(F_{N}, \boldsymbol{F}_{\boldsymbol{t}}, d\right)$ is, $g^{d}\left(F_{N}, \boldsymbol{F}_{\boldsymbol{t}}, d\right) \geq g^{f}\left(F_{N}, \boldsymbol{F}_{\boldsymbol{t}}, d\right)$ - $i$. e. damage takes place before fracture, apart from the limit case of a perfectly brittle interface $\left(F_{0}^{f}=F_{0}^{d}\right)$, where damage and fracture are concomitant.

Eventually, the damage evolution law is given by ( $\eta$ is a characteristic time):

$$
\dot{d}=\frac{1}{\eta}\left\langle\frac{g^{d}\left(F_{N}, \boldsymbol{F}_{\boldsymbol{t}}, d\right)}{F_{0}^{d}}\right\rangle H^{-}\left(-g^{f}\left(F_{N}, \boldsymbol{F}_{\boldsymbol{t}}, d\right)\right)+\left[\frac{1}{m}-d\right] \delta\left(g^{f}\left(F_{N}, \boldsymbol{F}_{\boldsymbol{t}}, d\right)\right)
$$


where $\langle$.$\rangle denotes the MacCauley brackets and H^{-}$is the modified Heaviside function $\left(H^{-}(0)=0\right)$. The Dirac distribution $\delta$ indicates that, as soon as $g^{f}\left(F_{N}, \boldsymbol{F}_{\boldsymbol{t}}, d\right)=0, \dot{d}$ is to be understood as a distribution derivative (i.e.d 'jumps' to its maximal value $1 / m)$.

\section{Illustrative examples}

\subsection{Tension}

Apart from the friction coefficient $\mu$, four material parameters have to be identified for the damaging interface model (see Section 2) to be fully defined: the softening parameter $m$; the damage yield $F_{0}^{d}$; the fracture yield $F_{0}^{f}=(1 / r) F_{0}^{d}(r \leq 1)$; the characteristic time $\eta$. The influence of each of these parameters on the damage evolution is here studied, considering a single interface (surface $S$ ) submitted to a simple tension such that $\boldsymbol{F}_{\boldsymbol{t}}=0$ and $\dot{\sigma}_{N}=\dot{F}_{N} / S=$ cst $>0$.

For a sake of convenience - and due to the fact that $t=\left(\sigma_{N} \sigma_{0}\right) /\left(\sigma_{0} \dot{\sigma}_{N}\right)$, where the damage yield stress $\sigma_{0}$ is given by $\sigma_{0}=F_{0}^{d} / S-d$ will be here considered as a function of $\sigma_{N} / \sigma_{0}$ instead of the time $t$. Thus, noticing that $g^{d}\left(\sigma_{N}, d\right)>0$ as soon as $\sigma_{N} / \sigma_{0}>1-m d_{0}$, Eqn. (4) can be rewritten (denoting by $d, N 0$ the first derivative of $d$ with respect to $\left.\sigma_{N} / \sigma_{0}\right)$ :

$$
\frac{\eta \dot{\sigma}_{N}}{\sigma_{0}} d,_{N 0}-m H\left(\frac{\sigma_{N}}{\sigma_{0}}-1+m d_{0}\right) d=\left(\frac{\sigma_{N}}{\sigma_{0}}-1\right) H\left(\frac{\sigma_{N}}{\sigma_{0}}-1+m d_{0}\right)
$$

with the initial condition $d\left(\sigma_{N} / \sigma_{0}=0\right)=d_{0}$. The exact solution of this equation reads (whenever $g^{f}\left(\sigma_{N}, d\right)=\sigma_{N}-\sigma_{f}(1-m d)<0$, where the fracture yield stress $\sigma_{f}$ is given by $\left.\sigma_{f}=F_{0}^{f} / S\right)$ :

$d\left(\frac{\sigma_{N}}{\sigma_{0}}\right)=d_{0}-H\left(\frac{\sigma_{N}}{\sigma_{0}}-1+m d_{0}\right)\left(\left(\frac{\eta \dot{\sigma}_{N}}{\sigma_{0} m^{2}}\right) \exp \left(\frac{m \sigma_{0}}{\eta \dot{\sigma}_{N}}\left(\frac{\sigma_{N}}{\sigma_{0}}-1+m d_{0}\right)\right)+\frac{1}{m}\left(1-\frac{\sigma_{N}}{\sigma_{0}}\right)-\frac{\eta \dot{\sigma}_{N}}{\sigma_{0} m^{2}}-d_{0}\right)$

Depending on different values of the material parameters, the different shapes of this solution are presented on Fig.1. Notice that, due to the fact that, in Eqn.(6), the material parameter $\eta$ and the loading parameter $\dot{\sigma}_{N}$ are systematically linked by their product, choice has been actually made to consider $\dot{\sigma}_{N}$ as a parameter and $\eta$ as a constant.

As shown on Fig.1, the main features of the damage evolution are:

- the loading rate $\dot{\sigma}_{N}$ (or, in an equivalent way, the inverse of the characteristic time $\eta$ ) acts on both the present damage $d$ - for an arbitrary given loading $\sigma_{N} / \sigma_{0}$, the greater $\dot{\sigma}_{N}$, the smaller $d-$ and the critical value of the damage $\left(d_{c}\right.$, such that $\left.g^{f}\left(\sigma_{N}, d_{c}\right)=0\right)$ - the greater $\dot{\sigma}_{N}$, the smaller $d_{c}$,

- the initial damage $d_{0}$ has influence on both the damage yield $\left(\sigma_{N 0}\right.$, such that $\left.g^{d}\left(\sigma_{N 0}, d_{0}\right)=0\right)$ - the greater $d_{0}$, the smaller $\sigma_{N 0}-$ and $d_{c}-$ the greater $d_{0}$, the greater $d_{c}$,

- the softening parameter $m$ immediatly gives the upper-bound of the damage range (since $d \in\left[0, \frac{1}{m}\right]$, see Section 2) and constrains the present damage $d$ - for an arbitrary given loading $\sigma_{N} / \sigma_{0}$, the greater $m$, the greater $d$,

- the ratio $r=F_{0}^{d} / F_{0}^{f}=\sigma_{0} / \sigma_{f} \leq 1$ acts only on the critical value of the damage - the greater $r$, the smaller $d_{c}$.

Another interesting result concerns the ultimate phase of the damage evolution, $i$. $e$. the fracture of the interface: the latter is not triggered by a critical value of the damage, a priori defined, but depends at he same times on the material parameters and on the loading parameter. From a modelling point of view, this is due to the fact that the damaging interface model is actually based on two yield surfaces, one for the damage, the other for the fracture; from a physical point of view, this result simply means that the fracture of the interface can be either 'brittle' (small values of $d_{c}$ ) $-e . g$. when submitted to high loading rates - or 'ductile' (great values of $d_{c}$ ) - e. $g$. for small values of the softening parameter.

\subsection{Compression}

We now consider an assembly of two-dimensionnal rigid solids (grains) - i.e. a two-dimensional granular medium - submitted to a compressive force $|T|$ in œdometric conditions (no lateral displacements). In the initial state, see Fig.2-a, the sample (initial heigth: $H=42 \mathrm{~cm}$; initial width: $W=48 \mathrm{~cm}$ ) is composed by 75 grains (diameters between 5 and $6 \mathrm{~cm}$ ), each of them being constituted by 60 to 70 particles (diameters $D^{p}$ between 5 and $6 \mathrm{~mm}$ ). More precisely, the numerical simulations involve 4980 particles. The loading $T$ 
is defined by a ramp (time rate $\dot{T}=c s t \neq 0)$ followed by a constant value $(\dot{T}=0)$, in order to highlight the creep like response of the granular medium. The axial strain is defined by $\epsilon=|U| / H$ where $U$ is the global displacement induced by $T$; the axial stress is denoted by $\sigma=|T| / e W$ where $e$ is the (unit) thickness of the sample. An other important parameter, denoted by $\nu$, is the ratio between the present number of broken interfaces and the initial number of cohesive contacts. Notice also that all the simulations were performed with the discrete element code LMGC90 (see $e . g$. [8]) and with $\mu=1, m=1, \eta=1 s$ and a time step $\Delta t=5 \cdot 10^{-4} \mathrm{~s}$.

As we have a time dependent damage model, the loading rate strongly influences the mechanical response of the sample. This is clearly shown on Fig.2-b, where $t$ is scaled by the loading characteristic time $t_{F}=F_{0}^{f} /|\dot{T}|$. For a given value of $\sigma_{0}$, Fig.2-c shows that $r$ influences the kinetics of the creep phase, while for a given value of $\sigma_{f}$, see Fig.2-d, this is the amplitude of the axial strain which is modified by $r$. Notice eventually that $\nu$ and $\epsilon$ evolves in the same way during the creep phase: the kinetics is mainly governed by the fracture of the interfaces.

\section{Conclusion}

Most of the structural failures are due to the pre-existence of various kinds of micro-defects (microcracks and/or microvoids) in the materials, which propagate and eventually coalesce in a macro-crack. The modelling of these propagation and coalescence is an important issue. The discrete approach presented here is intended as a step toward this issue. The proposed damaging interface model is based on a reduced set of five parameters. The illustrative examples seem to indicate that the numerical code in which the damaging model has been implemented is an efficient tool for simulating the initiation and the propagation of macro-cracks in rigid solids, including the time effect. Examples of applications clearly include dam engineering: rockfill material is characterized by delayed grain breakage under constant load. This is the main cause of the majority of post-constructive displacements observed in high rockfill dams, which can produce piping or cracking of the impervious element.

\section{Acknowledgements}

This project was sponsored by the Région Provence Alpes Côte d'Azur

\section{References}

[1] R. Deluzarche and B. Cambou, Discrete numerical modelling of rockfill dams, Int. J. Numer. Anal. Geomech., 30 (2006) 1075-1096.

[2] L.A. Oldecop and E.E. Alonso, Fundamentals of rockfill time-dependent behaviour, in: Juca, de Campos \& Marinho (Eds), Unsaturated Soils, 2002, pp. 793-798.

[3] M. Jean, The non-smooth contact dynamics method, Computer Methods in Applied Mechanic and Engineering, 177 (1999) 235-257.

[4] J.-J. Moreau, Unilateral contact and dry friction in finite freedom analysis, in: J.J Moreau et P.D Panagiotopoulos (Eds), Non Smooth Mechanics and Application, chapter CISM Courses and Lectures, Vol.302, Springer-Verlag, 1988, pp. 1-82.

[5] J.-Y. Delenne, M.S. El Youssoufi, F. Cherblanc, J.-C. Benet, Mechanical behaviour and failure of cohesive granular materials, Int. J. Numer. Anal. Geomech., 28 (2004) 1577-1594.

[6] J.-J. Marigo, Formulation d'une loi d'endommagement d'un matériau élastique, C. R. Acad. Sci. Paris, Ser. II 292 (1981) 1309-1312.

[7] L. Cangemi, M. Cocou and M. Raous, Adhesion and friction model for the fibre/matrix interface of a composite, in: A.B. Sabir, C. Bohatier, M.G. Fertis, G.T. Tsatsaronis, R.J. Krane, K.M. Abbott (Eds), Proc. Third Biennal Joint Conference on Engineering Systems, Design and Analysis (ESDA 96), Montpellier, Vol.1, 1996, pp. 157-163.

[8] B. Cambou and M. Jean, Micromécanique des matériaux granulaires, Hermès Science Publications, Paris, 2001. 

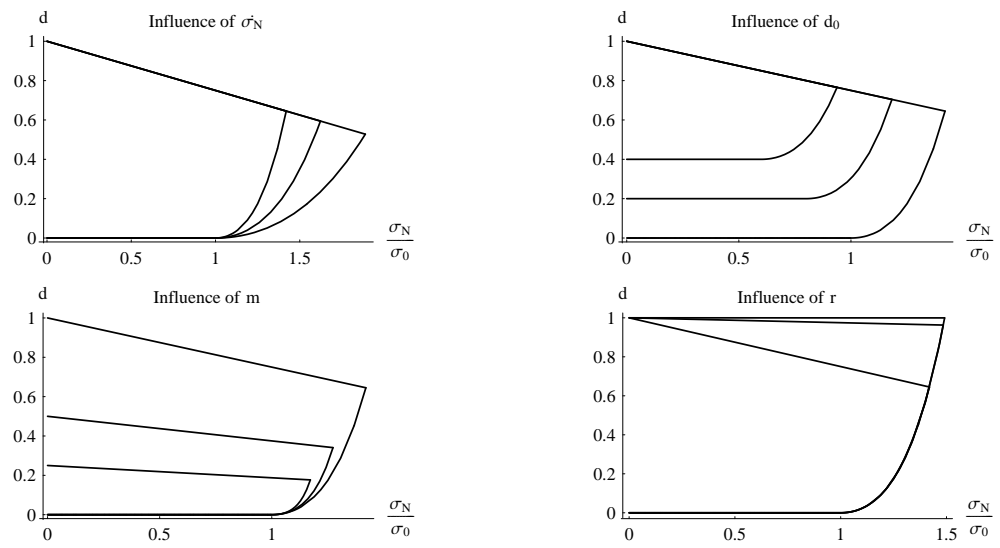

Figure 1: Simple tension of a single interface: influence of the loading and material parameters on the damage evolution. Notice that only the exponential part of each graph (ending in $d=d_{c}$ ) corresponds to a regular damage evolution: the linear part (ending on the $d$-axis to the maximum value of $d, 1 / m)$ is only an arbitrary representation of the damage jump $[d]=1 / m-d_{c}$, which leads to the fracture of the interface. Beyond $\sigma_{0}=0.9 \mathrm{MPa}$ and $\eta=0.1 \mathrm{~s}$, the reference parameters are: $\dot{\overline{\sigma_{N}}}=2.3 \mathrm{MPa}_{\mathrm{\sigma}^{-1}}, \overline{d_{0}}=0, \bar{m}=1, \bar{r}=0.25$ - 1a (up-left): influence of the loading rate, $\dot{\sigma}_{N}=$ $\dot{\overline{\sigma_{N}}}, 2 \dot{\overline{\sigma_{N}}}, 4 \dot{\overline{\sigma_{N}}}$; the greater $\dot{\sigma}_{N}$, the smaller $d_{c}-1 \mathrm{~b}$ (up-right): influence of the initial damage, $d_{0}=0,0.2,0.4-1 \mathrm{c}$ (down-left): influence of the softening parameter, $m=\bar{m}, 2 \bar{m}, 4 \bar{m}$; the greater $m$, the smaller $d_{c}-1 \mathrm{~d}$ (down-right): influence of the ratio $r=\sigma_{0} / \sigma_{f}, r=\bar{r}, 0.1 \bar{r}, 0.001 \bar{r}$; the greater $r$, the smaller $d_{c}$.
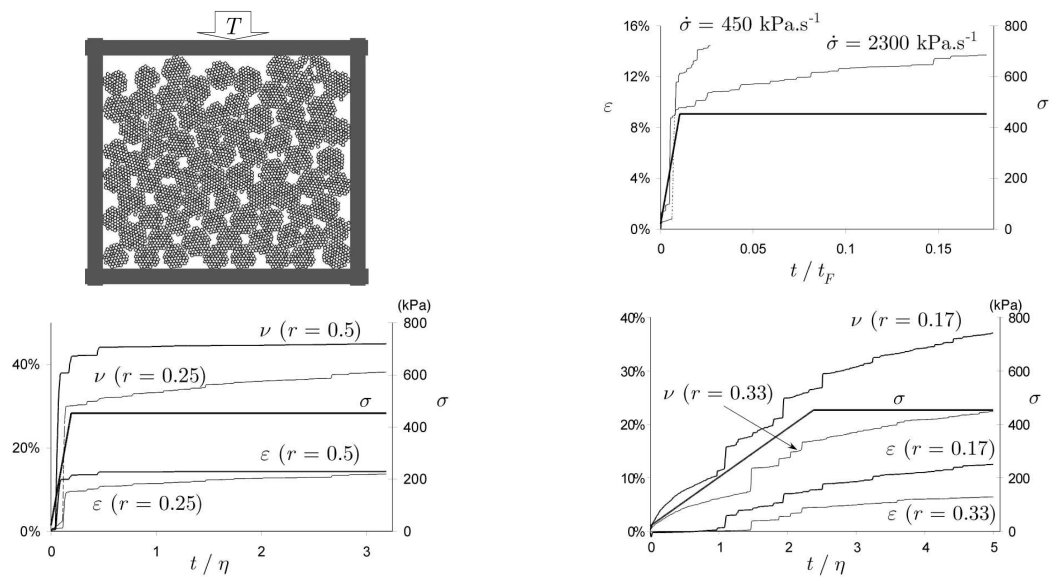

Figure 2: 2a (up-left): Sample composed by an assembly of 75 non 'glued' grains (initial heigth: $H=42 \mathrm{~cm}$; width: $W=48 \mathrm{~cm}$ ) and submitted to an œedometric loading ; each of the grains is composed of $\approx 65$ particles, initially 'glued' - $2 \mathrm{~b}$ (up-right): Axial strain $\epsilon=|U| / H$ versus dimensionless time $t / t_{F}$ for 2 loading rates ; $\sigma_{0}=900 \mathrm{kPa}, r=0.25-2 \mathrm{c}$ (bottom-left): Axial strain $\epsilon=|U| / H$ and ratio between the present number of broken interfaces and the initial number of cohesive contacts, $\nu$, versus dimensionless time $t / \eta ; \sigma_{0}=900 \mathrm{kPa} ; \dot{\sigma}=2300 \mathrm{kPa} . \mathrm{s}^{-1} ; r=0.25$ or 0.5 - 2d (bottom-right): Idem $2 \mathrm{c}$ apart from $\sigma_{f}=5500 \mathrm{kPa} ; \dot{\sigma}=180 \mathrm{kPa} . \mathrm{s}^{-1} ; r=0.17$ or 0.5 